\title{
Open Access für die Rechtswissenschaft: Pflicht oder Privatsache? Eine bibliothekarische Perspektive
}

\author{
Christian Mathieu*
}

Immer mehr Universitäten, Forschungseinrichtungen und Förderorganisationen bekennen sich zu dem Ziel, die Transformation des akademischen Kommunikationssystems unter dem Leitbild des freien Zugangs zu wissenschaftlichem Wissen mit Hilfe institutioneller Verpflichtungen - so genannter Open Access-Mandate zu beschleunigen. Disziplinäre Repositorien, die parallel zu den lokalen Open Access-Infrastrukturen von Hochschulen und Forschungseinrichtungen betrieben werden, stellen solche Arrangements allerdings durchaus vor strukturelle Herausforderungen bei der Akquise neuer Inhalte. Mit Blick auf dieses Spannungsverhältnis möchte der Beitrag die für das vorliegende Themenheft programmatische Leitfrage „Open Access für die Rechtswissenschaft: Pflicht oder Privatsache?" aus Sicht des Fachinformationsdiensts für internationale und interdisziplinäre Rechtsforschung zu beantworten versuchen und dabei einige Zukunftsperspektiven zum Strukturwandel der rechtswissenschaftlichen Publikationskultur skizzieren.

Aus Sicht der jüngst gegründeten cOAlitionS dürfte die programmatische Leitfrage der zweiten Tagung des sich für den freien Zugang zu juristischem Wissen engagierenden JurOA-Netzwerks „Open Access für die Rechtswissenschaft: Pflicht oder Privatsache? “ entschieden sein. ${ }^{1}$ Denn diese von Science Europe organisierte internationale Allianz von 16 staatlichen und privaten Forschungsfördereinrichtungen hat es sich zum Ziel gesetzt, die Transformation des wissenschaftlichen Publikationssystems durch eine konsequente Open Access-Politik zu befördern: So müssen ab Januar 2021 alle Veröffentlichungen aus Projekten mit Finanzierung durch eine Mitgliedsinstitution der cOAlitionS unmittelbar mit Erscheinen frei zugänglich und nachnutzbar sein - also nicht, wie es etwa die Open Access-Regularien, die so genannten Open Access-Mandate, der europäischen Forschungsrahmenprogramme gestatten, erst nach Ablauf einer maximal zwölfmonatigen Karenzzeit. ${ }^{2}$

Zwar dürften solche verpflichtenden Initiativen in Bibliotheken und anderen Informationsinfrastruktureinrichtungen in aller Regel ungeteilte Zustimmung finden, gelten doch möglichst strenge institutionelle Open Access-Mandate sowie entsprechend ausgestaltete Förderbestimmungen als probate Instrumente zur Beschleunigung des Strukturwandels der Wissenschaftskommunikation unter dem Leitbild

* Dr. Christian Mathieu ist Fachreferent in der Benutzungsabteilung der Staatsbibliothek zu Berlin.

1 http://www.juroa.de.

2 https://www.coalition-s.org/10-principles. 
des freien Wissenszugangs. ${ }^{3}$ Seiner prinzipiellen Open Access-Affinität zum Trotz nimmt sich aus der Perspektive des Fachinformationsdiensts für internationale und interdisziplinäre Rechtsforschung das Bild freilich ambivalent aus, weshalb auch der im Folgenden unternommene Versuch einer Antwort auf die eingangs angesprochene Leitfrage nach dem Königsweg zur Etablierung des Open Access-Publikationsmodells in den Rechtswissenschaften differenzierter ausfallen muss. Dabei resultiert der hier gewählte reduktionistische Ansatz, das Oppositionsverhältnis zwischen Pflicht und Privatsache als Vehikel für mehr juristischen Open Access vorrangig aus einer spezifischen Partikularsicht zu betrachten, aus dem Konzept der Frankfurter Tagung, eine Vielzahl von Einzelfacetten zu einem umfassenden Mosaik zusammenzufügen.

\section{A. Open Access als Handlungsfeld des Fachinformationsdiensts für internationale und interdisziplinäre Rechtsforschung}

Auftrag der von der Deutschen Forschungsgemeinschaft projektförmig geförderten Fachinformationsdienste für die Wissenschaft ist es, die Grundversorgung der Angehörigen aller Disziplinen durch Universitäts- und Seminarbibliotheken im Spitzenbereich zu komplementieren und insbesondere zentralen Zugriff auf vor Ort nicht verfügbare Spezialliteratur und forschungsrelevante Informationen zu ermöglichen. ${ }^{4}$ Für die Rechtswissenschaften übernimmt diese Aufgaben der an der Staatsbibliothek zu Berlin angesiedelte Fachinformationsdienst für internationale und interdisziplinäre Rechtsforschung, dessen profilprägende inhaltliche Fokussierung an den 2012 durch den Wissenschaftsrat skizzierten Perspektiven der Rechtswissenschaft in Deutschland orientiert ist - ein wissenschaftspolitisches Positionspapier, in dem im Wesentlichen für die Aufwertung der juristischen Grundlagenfächer, die Förderung der Interdisziplinarität rechtwissenschaftlicher Forschung sowie deren stärkere Internationalisierung plädiert wird. ${ }^{5}$

Neben einem personalisierten Fernleihservice sowie einem überregional zugänglichen virtuellen Lesesaal für in Deutschland bislang kaum verfügbare Datenbanken und elektronische Zeitschriften umfasst das Serviceportfolio des Fachinformationsdiensts für internationale und interdisziplinäre Rechtsforschung nicht zuletzt auch Beratungsangebote und technische Infrastrukturen für das wissenschaftliche Publizieren im Open Access. ${ }^{6}$ Denn als Einrichtung der Staatsbibliothek zu Berlin und

3 P. Vincent-Lamarre, J. Boivin, Y. Gargouri, V. Larivière, S. Harnad, Estimating Open Access Mandate Effectiveness: The MELIBEA Score, Journal of the Association for Information Science and Technology 2016, S. 2815.

4 Vgl. C. Kümmel, Nach den Sondersammelgebieten: Fachinformationen als forschungsnaher Service, Zeitschrift für Bibliothekswesen und Bibliographie 2013, S. 5.

5 Siehe dazu I. Vogel, C. Mathieu, Rechtswissenschaftliche Fachinformationsversorgung im Wandel: Zur Transformation des Sondersammelgebiets Recht in einen Fachinformationsdienst für internationale und interdisziplinäre Rechtsforschung, Recht, Bibliothek, Dokumentation 2014, S. 1.

6 Alle Dienstleistungen sind verfügbar via: https://vifa-recht.de. 
Projekt der Deutschen Forschungsgemeinschaft fühlt sich der Fachinformationsdienst dem Open Access-Gedanken verpflichtet, wie er in der von beiden Institutionen unterzeichneten und für die internationale Open Access-Bewegung konstitutiven Berliner Erklärung über den offenen Zugang zu wissenschaftlichem Wissen formuliert ist. ${ }^{7}$ Über seine zahlreichen Aktivitäten zur nachfragegetriebenen Retrodigitalisierung urheberrechtsfreier rechtswissenschaftlicher Forschungs- und Quellenliteratur hinaus ${ }^{8}$ - zumeist in Kooperation mit anderen Wissenschaftseinrichtungen oder juristischen Fachgesellschaften - betreibt der Fachinformationsdienst mit <intR $>2$ Dok ein disziplinäres Open Access-Repositorium für die Erst- und Zweitveröffentlichung von Texten, Datensammlungen und audio-visuellen Materialien zur internationalen und interdisziplinären Rechtsforschung. ${ }^{9}$ Im Unterschied zu institutionellen Repositorien, die ausschließlich den mit der jeweiligen Betreibereinrichtung affiliierten Personen zur Verfügung stehen, adressiert $<$ intR $>2$ Dok die Community der in internationaler bzw. interdisziplinärer Perspektive rechtswissenschaftlich Forschenden gleichermaßen. Genutzt wird diese Plattform vorrangig von Hochschulen und juristischen Fachgesellschaften ohne institutionelles Repositorium zur elektronischen Publikation ihrer Dissertationen und Schriftenreihen oder auch von dislozierten Forschungsverbünden wie dem Berliner Seminar Recht im Kontext zur öffentlichen Zugänglichmachung von Konferenzaufzeichnungen. Aufgrund der international standardkonformen technischen Implementierung von <intR $>^{2}$ Dok - deren Qualität wurde unlängst im Zuge eines Evaluierungsverfahrens zertifiziert - nehmen teilweise sogar Angehörige von Universitäten mit eigenen Open Access-Infrastrukturen diesen Service in Anspruch. Dabei erhöht vor allem seine Interoperabilität mit dem Forschungsinformationssystem OpenAIRE den Bedienkomfort des Repositoriums, werden auf diese Weise doch Projektgruppen mit EU-Förderung - unter ihnen Transnational Force of Law um Andreas Fischer-Lescano - in die Lage versetzt, ohne zusätzlichen Aufwand sowohl dem spätestens seit dem aktuellen Rahmenprogramm Horizon 2020 für den europäischen Forschungsraum verbindlichen Open Access-Mandat zu entsprechen als auch ihren Berichtspflichten über die im Kontext des betreffenden Vorhabens entstandenen Veröffentlichungen. ${ }^{10}$

Hoher Aufmerksamkeit erfreut sich $<$ intR $>^{2}$ Dok zudem von Seiten der expandierenden rechtswissenschaftlichen Blogosphäre: ${ }^{11}$ Namentlich sind es JuWissBlog,

7 https://openaccess.mpg.de/Berliner-Erklaerung.

8 I. Vogel, E. Schrecklinger, Retrospective Digitisation of Legal Sources in Germany, Legal Information Management 2014, S. 105.

9 https://intr2dok.vifa-recht.de.

10 https://www.openaire.eu.

11 Siehe H. Birkenkötter, M. Steinbeis, Rechtswissenschaftliche Blogs in Deutschland: zu Möglichkeiten und Grenzen eines neuen Formats in den Rechtswissenschaften, Jura: Juristische Ausbildung 2015, S. 23. 
Verfassungsblog und Völkerrechtsblog - diese zählen zu den angesehensten juristischen Wissenschaftsblogs in Deutschland -, die über $<$ intR $>2$ Dok internationalen Nachweis und dauerhafte Zitationsfähigkeit ihrer Inhalte sowie deren elektronische Langzeitarchivierung sicherstellen. Durch die Behandlung der Blogbeiträge als katalogisierungswürdiges und folglich auch mit konventionellen bibliothekarischen Rechercheinstrumenten auffindbares wissenschaftliches Publikationsformat trägt der Fachinformationsdienst für internationale und interdisziplinäre Rechtsforschung über den Zugewinn an weltweiter Sichtbarkeit hinaus dazu bei, symbolisches Kapital oder zumindest eine zusätzliche Seriositätsbürgschaft auf das noch junge Medium des Blogs und zugleich auf das Open Access-Paradigma zu übertragen, dem von Seiten der Rechtswissenschaften zumindest im deutschsprachigen Raum nach wie vor vergleichsweise zurückhaltend begegnet wird ${ }^{12}-$ im Gegensatz etwa zu den USA, bekennen sich dort doch die Law Schools zahlreicher Universitäten zu den Zielen des bereits 2008 formulierten Durham Statement on Open Access to Legal Scholarship. ${ }^{13}$

\section{B. Ambivalenzen von Open Access-Mandaten aus Sicht einer disziplinären Informationsinfrastruktureinrichtung}

Zwar stammt ein Großteil der am stärksten nachgefragten Inhalte von $<$ intR $>2$ Dok aus Projekten des aktuellen europäischen Forschungsrahmenprogramms, deren Ergebnisse sowie die diesen gegebenenfalls zugrundeliegenden Datensätze spätestens nach Ablauf einer Embargofrist von 12 Monaten im Open Access veröffentlicht sein müssen. Und auch die für den erfolgreichen Abschluss von Promotionsverfahren konstitutive Verpflichtung, Dissertationsschriften zu publizieren und damit dem Säurebad des jeweiligen Wissenschaftsdiskurses auszusetzen, kann als ein für den Fachinformationsdienst durchaus günstiges Mandat gelten, obschon Universitäten in aller Regel ihre Promovierenden zur Nutzung des lokalen institutionellen Repositoriums ermuntern, wenn nicht sogar verpflichten. Perspektivisch dürfte dieses Handlungsfeld zudem noch weiter an Bedeutung gewinnen, wird doch immer wieder der Zwang zur Open Access-Veröffentlichung von akademischen Qualifikationsschriften als Maßnahme zur Verhinderung von Plagiaten diskutiert. ${ }^{14}$

Demgegenüber stehen freilich zahlreiche Open Access-Reglements, die den Fachinformationsdienst für internationale und interdisziplinäre Rechtsforschung vor veritable Herausforderungen bei der Akquise neuer Inhalte stellen: So verlangt etwa

12 So A. Severin, M. Egger, M.P. Eve, D. Hürlimann, Discipline-Specific Open Access Publishing Practices and Barriers to Change: An Evidence-Based Review, F1000Research 2018, DOI: 10.12688/ f1000research.17328.1.

13 https://cyber.harvard.edu/publications/durhamstatement.

14 Vgl. E. Staudegger, Open-Access-Veröffentlichungspflicht für Dissertationen?: Eine rechtswissenschaftliche Untersuchung aus Anlass der Ergänzung von \$86 Abs 1 UG durch BGBlI 2017/129, ALJ 2018, S. 1 . 
die von der Universität Konstanz auf Grundlage des baden-württembergischen Hochschulgesetzes erlassene Satzung zur Ausübung des wissenschaftlichen Zweitveröffentlichungsrechts gemäß $\ 38$ Abs. 4 Urh $G$ von den Hochschulangehörigen, zu diesem Zweck ausschließlich das institutionelle Repositorium zu nutzen, ${ }^{15}$ obwohl die Landesnorm lediglich die Open Access-Publikation auf einem Repositorium vorsieht. ${ }^{16}$ Überdies hat es sogar den Anschein, als habe diese Initiative der prinzipiellen Akzeptanz des Open Access-Publikationsmodells von Seiten der Rechtswissenschaften einen Bärendienst erwiesen, war es doch just die juristische Fakultät der Universität Konstanz, die sich einer Verpflichtung zur Ausübung von Zweitveröffentlichungsrechten verweigern sollte. Zudem seien Zwangsmittel kaum mit dem Ideal des offenen Zugangs zu freiem Wissen vereinbar, wie der Sprecher des Fachbereichs Rechtswissenschaft der Konstanzer Universität an deren Rektor schreibt, zumal auch Zweifel an der Verfassungskonformität von Open AccessMandaten bestünden - ein Vorbehalt, zu dessen Klärung zuvor bereits die Deutsche Forschungsgemeinschaft ein Rechtsgutachten in Hinblick auf die Ausgestaltung ihrer Förderbedingungen in Auftrag gegeben hatte. ${ }^{17}$

"Unsere Kritik" - so Hans Theile in seiner angesprochenen Remonstration an den Konstanzer Rektor - „richtet sich nicht gegen die Idee von Open-Access selbst, wohl aber gegen den durch die Universität eingeschlagenen Weg, die Möglichkeit der Zweitveröffentlichung in ein auf diese Weise diskreditiertes Zwangsinstrument umzuwandeln. Angesichts der prinzipiellen Offenheit gegenüber der Open-Access-Idee verstört uns die Art und Weise, mit der sie an unserer Universität zwangsweise durchgesetzt werden soll, anstatt auf die individuelle Entscheidung der an ibr tätigen Wissenschaftlerinnen und Wissenschaftler zu vertrauen. " 18

Neben Satzungen steht mit dem System der leistungsorientierten Mittelzuweisung Rektoraten und Präsidien darüber hinaus noch ein weiterer, gerade an medizinführenden Universitäten verbreiteter Anreizmechanismus zur Beförderung des Open Access-Publikationsmodells zur Verfügung, der aus strukturellen Gründen aber ebenfalls institutionelle Repositorien privilegiert. Da mit der Auszahlung flexibler Gehaltsbestandteile nicht zuletzt auch die möglichst vollständige zentrale Dokumentation des institutionellen Publikationsaufkommens honoriert werden soll, verlieren für die Angehörigen der betreffenden Einrichtung externe disziplinäre Angebote aus Komfortgründen an Attraktivität, sind doch Hochschulbibliographien und Forschungsinformationssysteme häufig mit den lokalen Open Access-Infrastrukturen verkoppelt. Unter diesen Bedingungen wäre die Nutzung eines disziplinären Open Access-Repositoriums also mit Mehraufwand verbunden, von dessen

$15 \rrbracket 2$ Abs. 2 Amtliche Bekanntmachung der Universität Konstanz Nr. 90/2015.

$16 \$ 44$ Abs. 6 LHG B-W.

17 Siehe M. Fehling, Verfassungskonforme Ausgestaltung von DFG-Förderbedingungen zur Open-Access-Publikation, Ordnung der Wissenschaft 2014, S. 179.

18 Zitiert nach M. Löwisch, Konstanzer Juristenfakultät verweigert sich der Pflicht zur Zweitveröffentlichung, Ordnung der Wissenschaft 2016, S. 135 (135 f.). 
Leistung sich die Forschenden in aller Regel keinerlei Wettbewerbsvorteil versprechen. Strukturell benachteiligt sind disziplinäre Repositorien schließlich auch in Hinblick auf die Inanspruchnahme jener Zweitveröffentlichungsrechte, die aus den mit Unterstützung der Deutschen Forschungsgemeinschaft erworbenen Allianz-, FID- und Nationallizenzen für Datenbanken, elektronische Zeitschriften und EBooks resultieren. Gemäß den dabei verbindlichen Grundsätzen für den Erwerb DFG-geförderter überregionaler Lizenzen sind zwar Autorinnen und Autoren legitimiert, „ihre in den lizenzierten Zeitschriften erschienenen Artikel in der Regel in der durch den Verlag publizierten Form zeitnah in institutionelle oder disziplinspezifische Repositorien ihrer Wahl einzupflegen und im Open Access zugänglich zu machen "19 - ein Recht, das daneben auch den autorisierten Einrichtungen in Bezug auf die betreffenden Veröffentlichungen ihrer Angehörigen einzuräumen ist. Allerdings wird ein Szenario wohl wenig realistisch sein, in dem Universitäten und Forschungseinrichtungen sich der Mühe unterziehen, von besagter Open AccessKlausel privilegierte Zeitschriftenaufsätze zu identifizieren, um diese nicht dem eigenen Repositorium, sondern stattdessen externen disziplinären Infrastrukturen zur Verfügung zu stellen.

\section{C. „Move Prestige to Open Access“20 - Zukunftsperspektiven für das rechtswissenschaftliche Publikationssystem}

Ungleich schwerer als diese aus Partikularsicht skizzierten Ambivalenzen dürften unter den aktuellen Rahmenbedingungen zwei Einwände gegen eine Pflicht zur Publikation rechtswissenschaftlicher Forschungsbeiträge im Open Access wiegen jenseits der angesprochenen verfassungsrechtlichen Bedenken. ${ }^{21}$ So tragen Mandate, die alternativ zum genuinen Open Access auch die zeitverzögerte Zweitveröffentlichung von zuvor bereits an anderer Stelle erschienener Zeitschriftenaufsätze und Bücher erlauben, zwar zur freien Zugänglichkeit von wissenschaftlichem Wissen bei. Letztlich werden damit aber nur wenige Anreize zur Open Access-Transformation des etablierten subskriptionsbasierten Publikationssystems gesetzt, bleibt doch die herausgehobene Bedeutung der konventionellen Erstpublikation im wissenschaftlichen Fachdiskurs und insbesondere für den akademischen Reputationserwerb unberührt. Auf dem Feld der Rechtswissenschaften sollte der Effekt derartiger Mandate für die Durchsetzung von Open Access als Normalmodell der Wissenschaftskommunikation dabei besonders gering bleiben, als doch - wie auch

19 http://www.dfg.de/formulare/12_181/12_181_de.pdf, S. 9.

20 Harvard University, Faculty Advisory Council Memorandum on Journal Pricing, vom 17. April 2012.

21 Siehe dazu auch A. Peukert, Ein wissenschaftliches Kommunikationssystem ohne Verlage - zur rechtlichen Implementierung von Open Access als Goldstandard wissenschaftlichen Publizierens, in: M. Grünberger/S. Leible (Hrsg.), Die Kollision von Urheberrecht und Nutzerverhalten im Informationszeitalter, Tübingen 2014, S. 145. 
Horst-Peter Götting und Anne Lauber-Rönsberg betonen - nach wie vor nur wenige etablierte juristische Open Access-Journals existieren, deren Renommee als gleichrangig mit dem konkurrierender Abonnementszeitschriften anerkannt wird. ${ }^{22}$ In Verbindung mit dem insbesondere auf dem wissenschaftlichen Nachwuchs lastenden Druck, symbolisches Kapital durch Veröffentlichungen in möglichst reputierten Kontexten zu erwerben, steht gerade aber diese relative Leerstelle umgekehrt denn auch der Einführung genuinen Open Access favorisierender Mandate im Bereich der Rechtswissenschaften entgegen.

Zur Überwindung dieses Spannungsverhältnisses und damit letztlich auch der Polarität von Pflicht und Privatsache wäre insofern also ein systematischer Transfer wissenschaftlichen Prestiges auf Open Access-Publikationsformate erforderlich, für den auf dem Feld der rechtshistorischen Forschung sogar bereits ein Präzedenzfall existiert. So wurde 2012 die hochangesehene, vom Frankfurter Max-Planck-Institut für europäische Rechtsgeschichte herausgegebene und auch weiterhin im Verlag Vittorio Klostermann erscheinende Zeitschrift Rechtsgeschichte in den sofortigen Open Access überführt sowie in Entsprechung zu der auf diese Weise gewonnen Reichweite einer grundlegenden Internationalisierung unterzogen. ${ }^{23}$ Und auch die allen Angehörigen der Max-Planck-Gesellschaft inzwischen aus zentralen Mitteln eröffnete Möglichkeit, ihre in Kooperation mit dem vor allem im Bereich der Rechts- und Sozialwissenschaften profilierten Nomos-Verlag geplanten Buchprojekte als genuine Open Access-Veröffentlichungen zu realisieren, dürfte ganz erheblich zum Statusgewinn frei zugänglicher juristischer Publikationen beitragen. ${ }^{24}$ Freilich wird die u.a. auch von der cOAlitionS intendierte Transformation des bisherigen Geschäftsmodells von Verlagen - im Open Access-Paradigma sind die Kosten einer Veröffentlichung nicht mehr rezeptionsseitig von den Lesenden, sondern vielmehr produktionsseitig von den Schreibenden bzw. von Forschungseinrichtungen und Förderorganisationen zu tragen - in einer Publikationskultur vergleichsweise leicht fallen, in der die Leistung namhafter Druckkostenzuschüsse für Monographien und Sammelwerke als Standardverfahren etabliert ist. Da für Aufsätze in subskriptionsbasierten rechtswissenschaftlichen Zeitschriften demgegenüber aber häufig Honorare gezahlt werden, müssten selbst von Fachgesellschaften oder Forschungseinrichtungen finanzierte und daher von Publikationsgebühren befreite juristische Open Access-Journale ihre Autorinnen und Autoren für die entgangenen Einnahmen mit symbolischem Kapital oder anderen Wettbewerbsvorteilen entschädigen.

22 So H.-P. Götting, A. Lauber-Rönsberg, Open Access und Urheberrecht, Ordnung der Wissenschaft 2015, S. 137 (146).

23 http://rg.rg.mpg.de.

24 https://www.mpdl.mpg.de/ueber-uns/nachrichten.html. 
Auch für dieses Szenario findet sich im Orbit der Max-Planck-Gesellschaft ein Präzedenzfall, der angesichts des Prestiges ihrer juristischen Institute ohne weiteres auf die Rechtswissenschaften übertragbar ist. Angespielt ist damit auf das 2011 von Max-Planck-Gesellschaft, Wellcome Trust und Howard Hughes Medical Institute gegründete und demzufolge mit einem beträchtlichen Reputationsvorschuss ausgestattete lebenswissenschaftliche Open Access-Journal eLife, dessen rigoros vertretener Qualitätsanspruch in Verbindung mit einem initialen Verzicht auf die Erhebung von Publikationsgebühren die Zeitschrift rasch zu einem mit Nature und Science konkurrierenden Organ machen sollte, in dem sogar so aufsehenerregende Beiträge wie derjenige über die spektakuläre Entdeckung der neuen Hominidengattung des Homo naledi veröffentlicht werden. ${ }^{25}$

Um auf dem Feld der Rechtswissenschaften einem Publikationssystem zu seinem Durchbruch zu verhelfen, in dem die ebenbürtige Konkurrenz von konventionellen und offen zugänglichen Zeitschriften bzw. Schriftenreihen einerseits die Pflicht zum sofortigen Open Access durchsetzbar macht, anderseits aber Anreize für eine freiwillige private Entscheidung für dieses Veröffentlichungsmodell schafft, möchte der Fachinformationsdienst für internationale und interdisziplinäre Rechtsforschung künftig sein diesbezügliches Serviceportfolio weiter ausbauen. Unter dem doppelten Vorbehalt sowohl einer Fortführung des gegenwärtig im Auftrag der Deutschen Forschungsgemeinschaft evaluierten Förderprogramms der Fachinformationsdienste für die Wissenschaft als auch einer günstigen Begutachtung eines allfälligen Folgeantrags ist zum einen geplant, Finanzierungskonsortien für die Verlagspublikation von Dissertationen zur internationalen und interdisziplinären Rechtsforschung im genuinen Open Access zu organisieren. Zum anderen aber soll das disziplinäre, vor allem auf Zweitveröffentlichungen ausgelegte Repositorium $<$ intR $>^{2}$ Dok um für Forschende kostenfreie Hosting-Angebote von Open Journal Systems und Open Monograph Press als technische Infrastrukturen für die Publikation von Zeitschriften, Monographien und Schriftenreihen im sofortigen Open Access ergänzt werden - um Maßnahmen also, die im Einklang mit den Plänen der cOAlitionS stehen.

25 https://elifesciences.org. 